\title{
The Effect of Density Gradient on the Self-modulated Laser Wakefield Acceleration with Relativistic and Kinetic Effects
}

\author{
Seung Hoon Yoo*, Jaehoon $\mathrm{Kim}^{1}$, Jong Uk Kim¹, Ju Tae Seo ${ }^{2}$, and Sang June Hahn ${ }^{2+}$ \\ ${ }^{1}$ Advanced X-ray Medical System Research Group, Korea Electrotechnology- \\ Research Institute, Ansan 426-170, Korea \\ ${ }^{2}$ Department of Physics, Chung-Ang University, Seoul 156-756, Korea
}

(Received November 19, 2008 : revised March 8, 2009 : accepted March 9, 2009)

\begin{abstract}
The propagation of an intense laser pulse through an upward density-gradient plasma in a self-modulated laser wakefield acceleration (SM-LWFA) is investigated by using particle-in-cell (PIC) simulations. In the fully relativistic and kinetic PIC simulations, the relativistic and kinetic effects including Landau damping enhance the electron dephasing. This electron dephasing is the most important factor for limiting the energy of accelerated electrons. However, the electron dephasing, which is enhanced by relativistic and kinetic effects in the homogeneous plasma, can be forestalled through the detuning process arising from the longitudinal density gradient. Simulation results show that the detuning process can effectively maintain the coherence of the laser wake wave in the spatiotemporal wakefield pattern, hence considerable energy enhancement is achievable. The spatiotemporal profiles are analyzed for the detailed study on the relativistic and kinetic effects. In this paper, the optimum slope of the density gradient for increasing electron energy is presented for various laser intensities.
\end{abstract}

Keywords : Self-modulated laser wakefield acceleration, Density gradient, Relativistic effect, Kinetic effect, Electron dephasing

OCIS codes : (140.3460) Lasers; (290.5910) Scattering; stimulated Raman; (350.5400) Plasmas; (350.7420) Waves

\section{INTRODUCTION}

To generate quasi-monoenergetic short bunch electron beams, two different methods have been studied. One is the laser wakefield accelerator (LWFA) [1] and the other is the self-modulated laser wakefield accelerator (SM-LWFA) [2]. The difference between these two schemes is the ratio of laser pulse length, $\lambda_{L}=c \tau$ and plasma wavelength, $\lambda_{p}=2 \pi c / \omega_{p}$, where $\tau$ is the laser pulse duration, $c$ is the speed of light, and $\omega_{p}$ is the plasma frequency. In the SM-LWFA, the laser pulse duration is longer than the plasma wavelength, $\lambda_{L}>>$ $\lambda_{p}$. Due to the Raman instability and the envelope instability, the laser pulse envelope is modulated. The modulation length is equal to the plasma wavelength. In SM-LWFA, we can use high density plasmas

\footnotetext{
*Corresponding author: yoo731@keri.re.kr

${ }^{\dagger}$ Corresponding author: sjhahn@cau.ac.kr
}

because $\lambda_{p}$ depends on $n_{e}^{-1 / 2}$, where $n_{e}$ is the electron density. This means that we can generate the electron beam with a high current. Another good point of SMLWFA is the simple injection mechanism of background electrons. When the self-modulated laser wakefield is generated, some background electrons are self-injected into the acceleration phase. This is caused by wave breaking or Raman backscattering. Although the wave breaking does not occur, the particle injection can be performed by the slow beat wave of the Raman backscattered wave and the original pulse [3]. The selfinjected electrons are then trapped by the self-modulated laser wakefield and accelerated to a high energy. In this case, however, the injection occurs randomly in the acceleration phase. Therefore, the energy spread is naturally almost $100 \%$. Many experiments demonstrated electron acceleration in the self-modulated laser wakefield regime [4-7]. Recently, the quasi-monoenergetic electron beam was generated in the SM-LWFA using the pinhole-like 
collimator [8] and the ultra intense laser pulse [9]. Thus the SM-LWFA is a very promising method for the high-charge (nano-coulomb scale) electron beam generation.

To achieve higher electron energies in the SM-LWFA, it is necessary to propagate an intense laser pulse over a long distance in a plasma without disruption. But, there are strong instabilities and and electron dephasing problem due to the velocity difference between the electron beam and the wake wave. The fluid simulation study showed that the phase slippage due to the difference between the phase velocity of the wakefield and the group velocity of the laser can be forestalled through the upward density tapered channel [10] and detailed analyses including relativistic focusing [11] and the evolution of the laser envelope [12] were investigated. However, to observe the optimum condition for the experiment with a density gradient scheme, the ParticleIn-Cell (PIC) simulation study including relativistic and kinetic effects is needed.

In this paper, it is shown that the electron dephasing, which limits the energy of the accelerated electrons, is enhanced by the relativistic and kinetic effects including the Landau damping. And the optimum slopes of the density gradient for increasing average energy of the accelerated electrons are presented for various laser intensities.

\section{SIMULATION RESULTS}

In order to study the effect of upward density gradient plasma on energy enhancement of the accelerated electrons, we have performed one-dimensional (1D) PIC simulation by using XOOPIC code [13]. In this simulation, we have chosen the parameters for the self-trapping induced by the slow-phase-velocity beat waves from the Raman backscattering. The laser pulse with a wavelength $\lambda_{L}=$ $1 \mu \mathrm{m}$ and a pulse duration of $\tau_{L}=700 \mathrm{fs}$ has an intensity $(I)$ of from $1.4 \times 10^{18} \mathrm{~W} / \mathrm{cm}^{2}$ to $1.2 \times 10^{19} \mathrm{~W} / \mathrm{cm}^{2}$, which means dimensionless amplitude from (moderate relativistic case) to $a_{0}=3$ (strong relativistic case) (for $\lambda_{L}=1 \mu \mathrm{m}$, dimensionless amplitude can be expressed as $a_{0}=0.85 \times 10^{-9} \sqrt{I}$ ). In this work, to save the expensive simulation time, a moving window is used. The total simulation window, which moves at the speed of light, has a size of $500 \mu \mathrm{m}$ and 8000 grids. And the total simulation particle number is $3.2 \times 10^{5}$.

As shown in Fig. 1, two kinds of longitudinal plasma density profile are considered. Figure 1 (a) shows the uniform density case with a $2 \times 10^{19} \mathrm{~cm}^{-3}$ plasma density. Throughout the previous simulation work [14], the optimum slope of the density gradient turns out to be related to the gradient starting point of the density gradient. In this work, the starting point was determined through extensive simulation work for the various parameters of position and slope. The optimum cases of the moderate relativistic and strong relativistic cases are shown in Fig. 1(b). The optimum slopes are $22 \%$ per $100 \mu \mathrm{m}$ in the moderate case $\left(a_{0}=1\right)$ and $35 \%$ per $100 \mu \mathrm{m}$ in the strong case $\left(a_{0}=3\right)$. The optimum starting point is $500 \mu \mathrm{m}$ propagation after uniform plasma for both cases. In the simulation, the laser pulse duration is about 20 times larger than plasma wavelength, so the modulated region of laser pulse due to the Raman forward instability is generated only in the sub-part of the laser pulse. Figures 2(a) and (b) show the transverse electric fields (plasma wakefields) of the laser pulse after particle trapping for the moderate relativistic and strong relativistic cases respectively. The modulation occurs from the leading edge of the laser envelop and is convected backwards. In the strong relativistic case as shown in Fig. 2(b), the modulated region is located $100 \mu \mathrm{m}$ ahead in the front part of the laser pulse compared with the moderate case. This means that the laser envelope modulation is related to the laser intensity. Because the modulated region in the moderate case is located $100 \mu \mathrm{m}$ backward compared with the strong case, the optimum starting
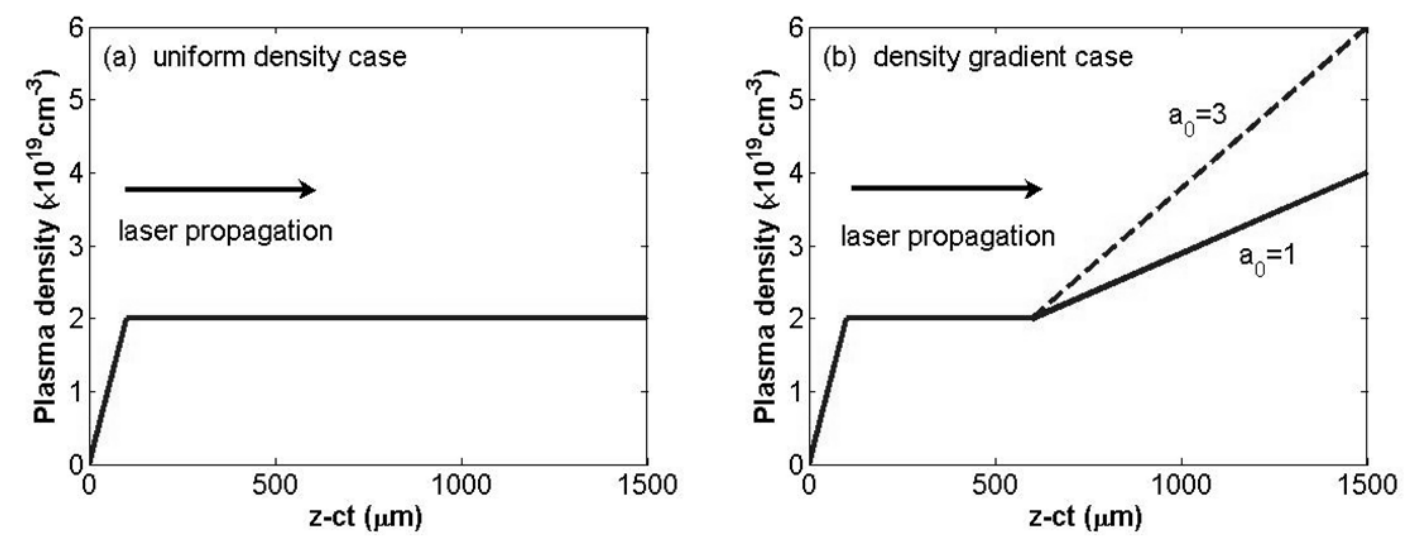

FIG. 1. Longitudinal plasma density profile. (a) uniform density case, (b) density gradient cases. 
point of the density gradient in the moderated case can be predicted $100 \mu \mathrm{m}$ earlier than the strong case. However, the self-injection does not occur for the case that the gradient starting point is $400 \mu \mathrm{m}$ propagation after uniform plasma. In this simulation, the self-trapping mechanism is induced by the Raman scattering. Thus the optimum position of gradient starting point is selected $500 \mu \mathrm{m}$ propagation point after uniform plasma for both cases. The corresponding electron phase spaces after particle trapping for the moderate relativistic and strong relativistic cases are presented in Fig. 2 (c) and (d) respectively.

In the one-dimensional limit, the electron dephasing length $\left(L_{d} \cong \lambda_{p}{ }^{3} / \lambda_{L}{ }^{2}\right)$ is defined as the length the electron must travel before it phase slips by one-half of a period with respect to the plasma wave [15]. In the fully relativistic and kinetic PIC simulations, the electron dephasing is enhanced by the relativistic effect and kinetic effect. Figure 3 show the spatio-temporal patterns of the plasma wakefield in the uniform cases ((a) $a_{0}=1$, (b) $a_{0}=3$ ) and in the density gradient cases ((c), (e), (g) $a_{0}=1,(\mathrm{~d}),(\mathrm{f}),(\mathrm{h}) a_{0}=3$ ). In the moderate relativistic cases ((a), (c), (e) and (g)), the modulated region occurs with the range of $160 \mu \mathrm{m}$ $240 \mu \mathrm{m}$ in the moving window. The selected modulated region is divided into two characteristic parts; front part and rear part of the modulated region. As shown in Fig. 3(a), in the front part of the modulated region, the phenomenon of the wake slippage is clearly observed within the moving window. After the dephasing point (after around 3.6ps), the pattern of the rear part begins to merge with the Landau damping. Thus, the temporal coherence of the wakefield between before and after the dephasing point is broken. However, in the optimum density gradient case ( $22 \%$ gradient case), as shown in Fig. 3(c), there is no noticeable merged pattern and the modulation pattern seems to be very regular, which shows that the wake slippage is forestalled. In the strong relativistic cases ((b), (d), (f) and (h)), the modulated region occurs with the range of $250 \mu \mathrm{m}$ -
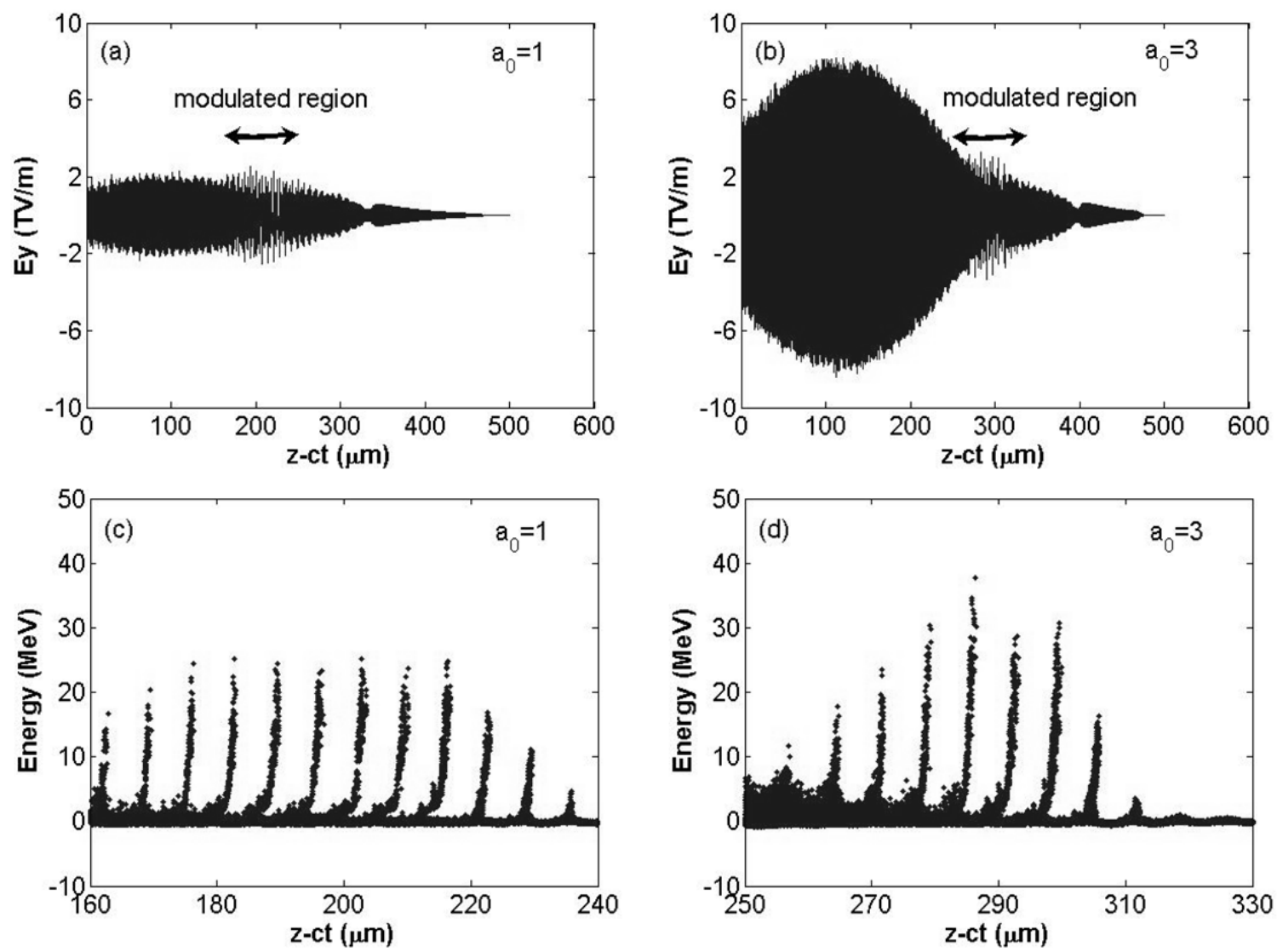

FIG. 2. Dynamics of electron trapping at before dephasing point.

(a) Transverse electric field (plasma wakefield) for $a_{0}=1$ at $\mathrm{t}=3.5 \mathrm{ps}$.

(b) Transverse electric field (plasma wakefield) for $a_{0}=3$ at $\mathrm{t}=3.5 \mathrm{ps}$.

(c) Electron momentum space for $a_{0}=1$ at $\mathrm{t}=2.9 \mathrm{ps}$.

(d) Electron momentum space for $a_{0}=3 \mathrm{at} \mathrm{t}=2.9 \mathrm{ps}$ 
$330 \mu \mathrm{m}$ in the moving window. Figure 3 (b) shows that the wake slippage is more enhanced than for the moderated case (See Fig. 3 (a).) by the relativistic effect in the front part. Also the merged pattern
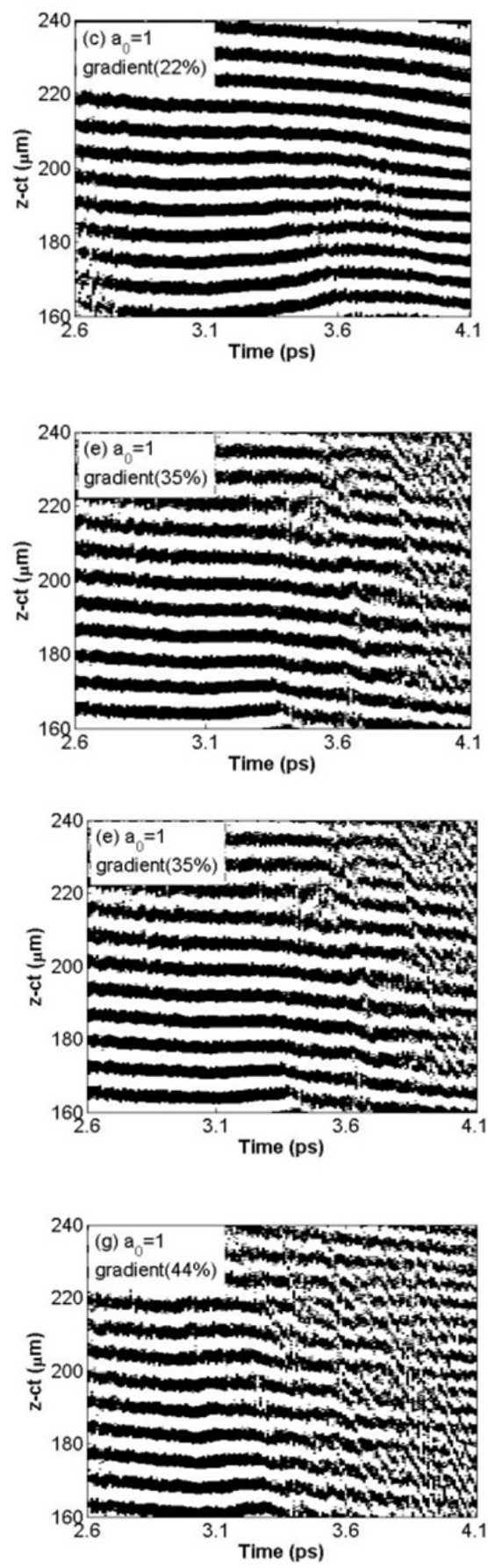

induced by the Landau damping occurs in the rear part. These figures (Fig. 3 (a) and (b)) show that the relativistic effects induced from the more intense laser pulse enhance the electron dephasing. This breaking of
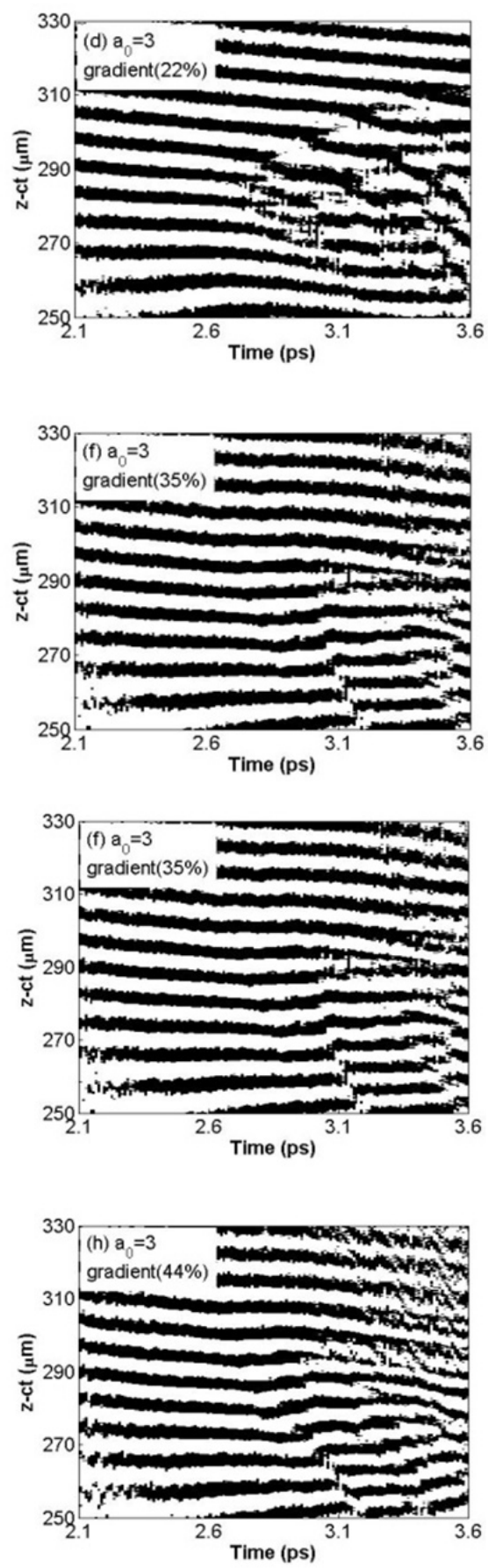

FIG. 3. Spatio-temporal pattern of plasma wakefiled.

(a) Uniform density case for $a_{0}=1$.

(b) Uniform density case for $a_{0}=3$.

(c), (e), (g) Density gradient cases for $a_{0}=1$ with various gradient slopes.

(d), (f), (h) Density gradient cases for $a_{0}=3$ with various gradient slopes. 
the temporal coherence is compensated with the more severe optimum slope of density gradient (35\% gradient case) as shown in Fig. 3(f). The modulation pattern seems to be nearly regular. Figure $3(\mathrm{e}),(\mathrm{g})$ and (h) shows that the plasma wakefields have weaker fields and are not generated regularly. The energy of accelerated electrons is limited by the irregular plasma wakefields with the increasing kinetic effect in the too stiff density gradient regime.

Figures 4(a) and (b) show the average energies of the accelerated electrons over $5 \mathrm{MeV}$ for the case of $a_{0}=1$ (moderated case) and $a_{0}=3$ (strong case). The uniform density case and various gradient cases are presented. In the both cases (moderate and strong cases), the average energies of accelerated electrons are saturated after electron dephasing in the uniform and gradient density cases. However, in the optimum density gradient for each case (The $22 \%$ density gradient is the optimum in the moderate case and the $35 \%$ density gradient is

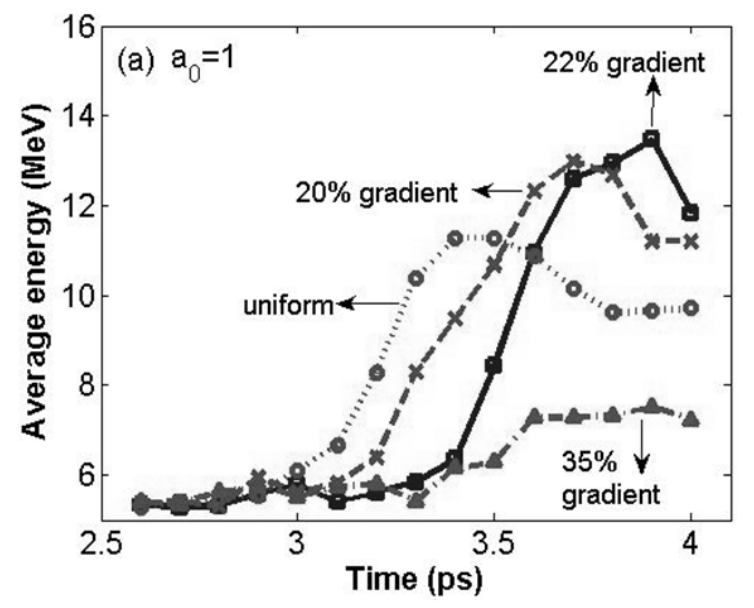

the optimum in the strong case.), the average energies increase about $25 \%$ compared to the uniform density cases with the forestalled dephasing point. This is a significant amount of energy enhancement in the SM-LWFA. In this work, the infinite plasma density to the laser propagation direction is used and the laser propagates through the plasma for a long time. Hence, the accelerated electrons are dephased after the dephasing point for all cases. The simulation results show that the energy enhancement of the accelerated electrons with a forestalled dephasing point can be obtained using the optimum density gradient. In the laser driven electron accelerator with a gas nozzle, the interaction length of the nozzle should be comparable to the dephasing length for obtaining the electron beam before dephasing. These simulation results show that the optimal slope of the density gradient and determine the suitable interaction length of the nozzle before dephasing in experimental plans. Also, in this simulation, the 700fs

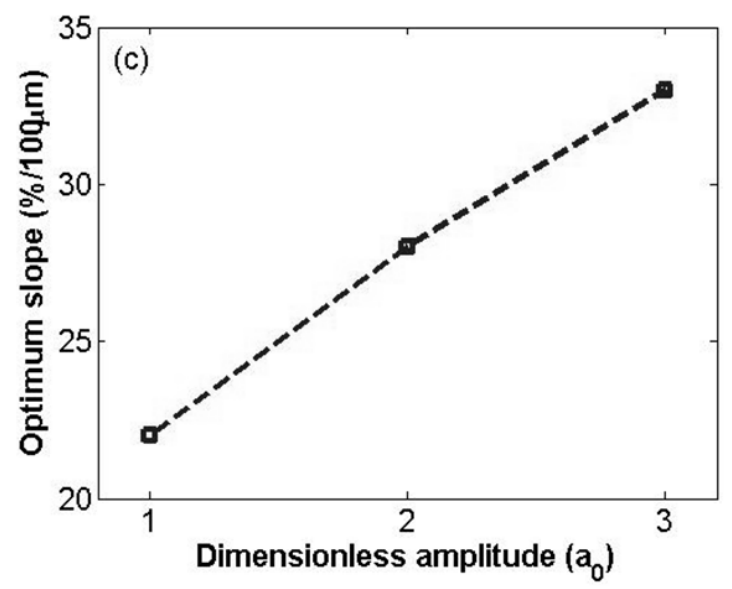

FIG. 4. Average energy of the accelerate electrons over $5 \mathrm{MeV}$ in the modulated region and scaling of the density gradient optimum slope.

(a) Average energy in the uniform and various gradient density cases for $a_{0}=1$,

(b) For $a_{0}=3$, and

(c) Scaling of the density gradient optimum slope for the laser intensity 
duration laser pulse is propagating the density gradient system. The gradient starting point is inside the modulated region not the leading edge of the laser pulse. Because the leading edge of the laser pulse is going through the different plasma density, there is dynamical delay between uniform and gradient cases. The scaling of the optimum slope for the laser intensity is shown in Fig. 4(c). It shows that the scaling of the optimum slope increases linearly from $a_{0}=1$ to $a_{0}=3$.

\section{DISCUSSION AND CONCLUSION}

In this paper, we have shown that the electron dephasing induced from the wake slippage is enhanced by the relativistic and kinetic effects. This electron dephasing can be forestalled using a density gradient scheme. Throughout the intensive simulation work for the various parameters, the optimum slopes of density gradient are determined for both cases. In the strong relativistic case, more severe slope is needed compared to the moderate relativistic case, however the starting point of the density gradient is almost the same for both cases. This is due to the self-injection mechanism in the SM-LWFA. Therefore, it is shown that the average energies of the accelerated electrons in the modulated region with optimum density gradient increase significantly due to the reduced dephasing effect.

\section{ACKNOWLEDGMENT}

This work was supported in part by the Ministry of Knowledge and Economy of Korea through the Ultrashort Quantum Beam Facility Program.

\section{REFERENCES}

1. T. Tajima and J. M. Dawson, "Laser electron accelerator,” Phys. Rev. Lett. 43, 267-270 (1979).

2. J. Krall, A. Ting, E. Esarey, and P. Sprangle, "Enhanced acceleration in a self-modulated-laser wake-field accelerator," Phys. Rev. E 48, 2157-2161 (1993).

3. C. I. Moore, A. Ting, K. Krushelnick, E. Esarey, R. F. Hubbard, B. Hafizi, H. R. Burris, C. Manka, and P. Sprangle, "Electron trapping in self-modulated laser wakefields by raman backscatter,” Phys. Rev. Lett. 79, 3909-3912 (1997).
4. K. Nakajima, D. Fisher, T. Kawakubo, H. Nakanishi, A. Ogata, Y. Kato, Y. Kitagawa, R. Kodama, K. Mima, H. Shiraga, K. Suzuki, K. Yamakawa, T. Zhang, Y. Sakawa, T. Shoji, Y. Nishida, N. Yugami, M. Downer, and T. Tajima, "Observation of ultrahigh gradient electron acceleration by self-modulated intense short laser pulse," Phys. Rev. Lett. 74, 4428-4431 (1995).

5. A. Ting, K. Krushelnick, C. I. Moore, H. R. Burris, E. Esarey, J. Krall, and P. Sprangle, "Temporal evolution of self-modulated laser wakefields measured by coherent Thomson scattering," Phys. Rev. Lett. 77, 5377-5380 (1996).

6. A. Modena, Z. Najmudin, A. E. Dangor, C. E. Clayton, K. A. Marsh, C. Joshi, V. Malka, C. B. Darrow, C. Danson, D. Neely, and F. N. Walsh, "Electron acceleration from the wavebreaking of relativistic plasma waves," Nature (London) 337, 606-608 (1996).

7. D. Umstadter, S.-Y. Chen, A. Maksimchuk, G. Mourou, and R. Wagner, "Nonlinear optics in relativistic plasmas and laser wake field acceleration of electrons," Science 273, 472-475 (1996).

8. N. Hafz, M. S. Hur, G. H. Kim, C. Kim, I. S. Ko, and H. Suk, "Quasimonoenergetic electron beam generation by using a pinholike collimator in a self-modulated laser wakefield acceleration," Phys. Rev. E 73, 016405-1-7 (2006).

9. B. Hiding, K.-U. Amthor, B. Liesfeld, H. Schwoere, S. Karsch, M. Geissler, L. Veisz, K. Schmid, J. G. Gallacher, S. P. Jamison, D. Jaroszynski, G. Pretzler, and R. Sauerbrey, "Generation of quasimonoenergetic electron bunches with 80-fs laser pulse,” Phys. Rev. Lett. 96, 105004-1-4 (2006).

10. J. R. Penano, B. Hafizi, P. Sprangle, R. F. Hubbard, and A. Ting, "Raman forward scattering and self-modulation of laser pulses in tapered plasma channels," Phys. Rev. E 66, 036402-1-13 (2002).

11. B. Hafizi, A. Ting, R. F. Hubbard, P. Sprangle, and J. R. Penano, "Relativistic effects on intense laser beam propagation in plasma channels," Phys. Plasmas 10, 1483-1491 (2003).

12. P. Jha, R. G. Singh, A. K. Upadhyaya, and R. K. Mishra, "Propagation of an intense laser beam in a tapered plasma channel," Phys. Plasmas 15, 033101-1-6 (2008).

13. J. P. Verboncoeur, A. B. Langdon, and N. T. Gladd, "An object-oriented electromagnetic PIC code," Comp. Phys. Comm. 87, 199-211 (1995).

14. S. H. Yoo, S. J. Hahn, M. S. Hur, H. Jang, I. Hwang, J. H. Kim, and H. Suk, "Energy enhancement of the self-modulated laser wakefield acceleration by using the plasma density gradient,” J. Korean. Phys. Soc. 51, 402-408 (2007).

15. E. Esarey, P. Sprangle, J. Krall, and A. Ting, "Overview of plasma-based accelerator concepts," IEEE Trans. Plasma Sci. 24, 252-288 (1996). 